Joint association between education and polygenic risk score for incident coronary heart disease events : a longitudinal population-based study of 26203 men and women

\title{
Martikainen, Pekka
}

2021-07

Martikainen, P, Korhonen, K, Jelenkovic , A, Lahtinen , H , Havulinna , A, Ripatti , S , Borodulin , K , Salomaa , V , Smith , G D \& Silventoinen , K 2021 , ' Joint association between education and polygenic risk score for incident coronary heart disease events : a longitudinal population-based study of 26203 men and women ' , Journal of Epidemiology \& Community Health , vol. 75 , no. 7 , pp. 651-657 . https://doi.org/10.1136/jech-2020-214358

http://hdl.handle.net/10138/337043

https://doi.org/10.1136/jech-2020-214358

cc_by_nc_nd

acceptedVersion

Downloaded from Helda, University of Helsinki institutional repository.

This is an electronic reprint of the original article.

This reprint may differ from the original in pagination and typographic detail.

Please cite the original version. 
The joint association between education and polygenic risk score for incident coronary heart disease events: a longitudinal population-based study of 26203 men and women

Pekka Martikainen $^{1,2,3}$, Kaarina Korhonen ${ }^{1}$, Aline Jelenkovic ${ }^{4,5}$, Hannu Lahtinen ${ }^{1}$, Aki S.

Havulinna $^{6,7}$, Samuli Ripatti ${ }^{5,7,8}$, Katja Borodulin ${ }^{6,9}$, Veikko Salomaa ${ }^{6}$, George Davey Smith ${ }^{10}$, Karri Silventoinen $^{1}$

1. University of Helsinki Faculty of Social Sciences, Population Research Unit, Finland, 2.

Stockholm University, Department of Public Health Sciences, Sweden, 3. Max-Planck-Institute for Demographic Research, Germany, 4. University of the Basque Country, Department of Physiology, Spain, 5. University of Helsinki Faculty of Medicine, Department of Public Health, Finland, 6.

National Institute for Health and Welfare, Department of Public Health Solutions, Finland, 7. Institute for Molecular Medicine Finland, Finland, 8. Broad Institute of MIT and Harvard, Cambridge, MA, USA, 9. Age Institute, Finland, 10. Medical Research Council Integrative Epidemiology Unit, Bristol Medical School, University of Bristol, UK.

Sources of funding: This work was supported by grants 308247 and 294861 from the Academy of Finland, HORIZON 2020 innovation action award number 667661 from the European Commission (project MINDMAP) and the Finnish Foundation for Cardiovascular Research.

Corresponding author:

Pekka Martikainen. Mailing address: University of Helsinki Faculty of Social Sciences, Population Research Unit, Unioninkatu 35 (P.O. Box 54), 00014 University of Helsinki, Finland; Tel.: +35844-286 1946; E-mail: pekka.martikainen@helsinki.fi

Keywords: social epidemiology, gene environment interactions, education, coronary heart disease Word count: 3200 
The joint association between education and polygenic risk score for incident coronary heart disease events: a longitudinal population-based study of 26203 men and women

\begin{abstract}
Background: Genetic vulnerability to coronary heart disease (CHD) is well established, but little is known whether these effects are mediated or modified by equally well-established social determinants of CHD. We estimate the joint associations of the polygenetic risk score (PRS) for CHD and education on CHD events.
\end{abstract}

Methods: The data are from the 1992, 1997, 2002, 2007 and 2012 surveys of the population-based FINRISK study including measures of social, behavioural and metabolic factors, and genome wide genotypes ( $\mathrm{N}=26$ 203). Follow-up of fatal and non-fatal incident $\mathrm{CHD}$ events $(\mathrm{N}=2063)$ was based on nationwide registers.

Results: Allowing for age, sex, study year, region of residence, study batch and principal components, those in the highest quartile of PRS for CHD had strongly increased risk of CHD events compared to the lowest quartile (HR=2.26; 95\% confidence interval: 1.97, 2.59); associations were also observed for low education $(\mathrm{HR}=1.58 ; 95 \%$ confidence interval: $1.32,1.89)$. These effects were largely independent of each other. Adjustment for baseline smoking, alcohol use, body mass index, HDL and total cholesterol, blood pressure and diabetes attenuated the PRS associations by $10 \%$ and the education associations by $50 \%$. We do not find strong evidence of interactions between PRS and education.

Conclusions: PRS and education predict CHD events, and these associations are independent of each other. Both can improve CHD prediction beyond behavioural risks. The results imply that observational studies that do not have information on genetic risk factors for CHD do not provide confounded estimates for the association between education and CHD. 
Keywords: Education, Polygenic risk, Interaction, Coronary heart disease

What is already known on this subject?

- Both socio-economic and genetic factors are associated with coronary heart disease (CHD).

- However, little is know with respect to whether the effects of genetic factors on CHD are mediated or modified by socio-economic factors.

What this study adds?

- Polygenic risk score for CHD and education are strongly and independently associated with CHD events in a representative sample of 26203 Finns.

- The CHD risk score and education are only modestly correlated with each other, and the effects of polygenic risk score for CHD on disease events are only weakly mediated or modified by education.

- The association between education and incident CHD events is only weakly confounded by the genetic risk of CHD. 


\section{INTRODUCTION}

Coronary heart disease (CHD) is the leading cause of death in high income countries (1). Especially in the northern part of Europe and the US, it also contributes substantially to socio-economic differences in mortality (2). Differences in CHD incidence and mortality have been found using several indicators of socio-economic position such as education (3), occupation-based social position (4) and income (5). These associations are at least partly mediated by the risk factors of CHD, and socio-economic differences have been found in CHD risk factors such as obesity (6) as well as smoking, blood pressure, glucose and lipid levels (7).

Genetic factors also affect the risk of CHD and its risk factors. Previous twin studies estimate that around $70-80 \%$ of the variation of body mass index (BMI) $(8,9), 50-60 \%$ of the variation of blood pressure (10), and 40-60\% of the variation of blood lipids (11) in adulthood can be explained by genetic differences between individuals. Studies on the heritability of CHD incidence on twins are relatively rare (12), but a Swedish study found that the heritability of CHD mortality is $57 \%$ in men and $38 \%$ in women suggesting that a clear genetic component can be found for CHD incidence (13). This finding is supported by higher concordance rates of CHD mortality in mono- than in dizygotic twins in a pooled study of Nordic twin registers (14). More recent GWA studies indicate a SNP heritability of around $15-25 \%$ for major CHD events $(15,16)$.

Genome wide association (GWA) studies allow to identify loci associated with increased CHD risk and to create polygenetic risk scores (PRS) for predicting CHD. Through the accumulation of known genetic polymorphisms, the variation explained by genetic factors has increased: a GWA metaanalytic study of CHD identified tens of loci associated with CHD risk (17) and a large consortium using genomic scans of nearly 200000 individuals found 62 loci explaining 10-15\% of the variation in key indicators of blood lipids (18). Also BMI is highly polygenic with those in the highest decile of risk having a 25-fold risk of severe obesity in middle age (19). An early genetic risk score of 13 
known loci also predicted cardiovascular disease incidence in the Framingham Hart Study (hazard ratio $=1.05$ per allele adjusted for traditional risk factors) (20). More recent analyses of PRS on incident CHD events in the FINRISK and Framingham Heart Study cohorts indicate an odds ratio of $1.74(95 \%$ CI: $1.61,1.89)$ per standard deviation (SD) and 1.28 (95\% CI: 1.17, 1.41) per SD respectively (21).

It is, however, unclear how much environmental factors modify the effects of these PRSs. For example, prior studies have found that the polygenetic risk scores for BMI are more strongly associated with BMI in physically inactive persons than in active persons (22); results consistent with previous twin study findings (23). Thus, physical activity seems to suppress the effect of genetic variants predisposing to obesity. Similar interactions have been observed for other types of obesogenic environments and social deprivation $(24,25)$. However, little is still known about whether social factors may modify the genetic risk of CHD.

We assessed the following research questions: (i) is genetic predisposition for CHD associated with CHD events through its association with education; (ii) is genetic predisposition for CHD more strongly associated with CHD events in people with lower education; and (iii) are these effects mediated through established CHD behavioural and metabolic risk factors? The analyses are based on the FINRISK Study (26), which includes measures of key behavioural and metabolic risk factors of CHD, register based follow-up of incident CHD events, various measures of socio-economic circumstances and PRS for CHD ( $\mathrm{N}=26203)$.

\section{DATA AND METHODS}

\section{Participants}

We used the FINRISK risk factor surveys carried out by The Finnish Institute for Health and Welfare (26) in 1992, 1997, 2002, 2007 and 2012 in North Karelia, Northern Savonia, Turku and Loimaa, Lapland (in 2002), the capital area including cities of Helsinki and Vantaa and the provinces of 
Northern Ostrobothnia and Kainuu. The data represent the populations in the study regions well. For each survey, an independent random sample was drawn from the national population register stratified by sex and 10-year age group from the population aged 25-74 years separately for each survey area. For the 1992 survey, the sample drawn was aged 25-64 years. The baseline varies for subjects participating in the five different survey collection points. In the rare cases that a respondent had participated in more than one survey, only the first participation was considered in this study. Participation rates varied from year to year, showing a downward trend with rates about $77 \%$ in 1992 and around $60 \%$ in the most recent survey. To ensure comparable data on risk factor trends, the methodology of the surveys has been kept as similar as possible. Ethical approval has been obtained according to required procedures over the study years. The Coordinating Ethics Committee of the Helsinki and Uusimaa Hospital District approved the study, which followed the declaration of Helsinki.

The surveys included 1) a self-administered questionnaire, 2) physical measurements and 3) blood samples. The questionnaire, together with an invitation to the health examination, was sent by mail to all participants. In addition, information from the cause of death register, hospital discharge register, prescribed drug purchase and drug reimbursement registers were linked with the sample.

We used a PRS for CHD based on 49310 single-nucleotide polymorphisms (SNP), developed in multiple cohorts including the FINRISK and Framingham Heart Studies (19). We obtained the GWAS data for FINRISK participants through several study projects using different GWAS chips and therefore adjusted for the GWAS batch as a factor variable. We adjusted for the ten first principal components derived from the genome wide data to control possible population stratification. We imputed the GWAS data using 1000 genomes, phase 3 reference panel. The PRS was calculated by adding numbers of risk alleles and using the logartihmized odds ratios as relative SNP weights as presented in Abraham et al (21). We validated our findings using an alternative PRS for CHD from 
Tada et al (27). Education was based on the highest attained education level reported in the questionnaire: tertiary education, high school, vocational training and basic education.

Behavioural and metabolic risk factors including smoking, alcohol use, BMI, blood pressure, cholesterol, and diabetes were incorporated as potential mediators. Smoking and alcohol use were assessed by structured questions in the self-administered questionnaire. Participants were classified as current smokers, former smokers and never smokers. Former smokers had smoked regularly, but quit at least 6 months before the survey. Based on detailed information on the amount of different types of alcoholic beverages during the past week, participants were classified as non-drinkers, low moderate drinkers (<35 g ethanol per week), high moderate drinkers (35-100 g ethanol per week) and heavy drinkers (>100 g ethanol per week).

Blood pressure as well as height and weight were measured in the physical examination. Systolic and diastolic blood pressures were measured twice or three times, and the mean of these measures was used in this study. BMI was calculated as weight in kilogrammes divided by squared height in metres. Total and HDL cholesterol were determined from frozen serum samples at the central laboratory of the Finnish Institute for Health and Welfare. We used systolic and diastolic blood pressures, BMI and total and HDL cholesterol as continuous variables in all analyses. Prevalent diabetes at the time of the survey was identified from the hospital discharge register (ICD-8/9 code 250 and ICD-10 codes E10-14), and the records of prescription drug purchases (ATC class A10) and entitlement to special reimbursement for diabetes medication (Finnish disease code 103 for diabetes mellitus).

Altogether, 27284 participants had genotypes available for PRS calculation. Those with pre-baseline CHD ( $n=636)$ and with missing information on any of the covariates $(n=365)$ were excluded from the analytic sample. Thus, we analysed 26203 participants free from CHD at baseline and followed for CHD death or hospitalisation from the time of the survey to the end of 2015. Censoring was at the end of follow-up or death from other causes than CHD; emigration was negligible. CHD event was defined for non-fatal cases as the International Classification of Diseases (ICD) $9^{\text {th }}$ revision codes 
410 and 4110 (years $1992-1995$ ) and $10^{\text {th }}$ revision codes I200, I21 or I22 (1996-2015) and NOMESCO codes for coronary artery bypass graft (CABG) or percutaneous coronary intervention (PCI) in the hospital discharge register, and for fatal cases as ICD-9 codes 410-414 and 798 (not 7980A) and ICD-10 codes I20-25, I46, R96 and R98 in the causes of death register. Fatal and nonfatal CHD events in the Finnish hospital discharge and cause of death registers are valid indicators when compared with the FINMONICA register data; with a sensitivity of $83 \%$ and a positive predictive value of $90 \%$. Only $1-4 \%$ of cases classified as MI or CHD in the FINMONICA register had ICD codes unrelated to CHD in the hospital discharge and cause of death registers (28). During the mean follow-up of 13.8 years, we observed 2063 incident CHD events.

\section{Statistical analyses}

The associations between PRS quartiles, education and incident CHD events were estimated with Cox proportional hazard models, using age as the time scale. To avoid small number of observations, we combined men and women for these analyses and adjusted for sex. Sex specific analyses are available in Supplementary table 1. The results are highly similar between men and women. Visual inspection of log-log plots implied that the proportional-hazards assumption was not violated. In model 0 , hazard ratios (HR) and their 95\% confidence intervals (CI) for incident CHD events were estimated separately by PRS quartile and education, controlling for sex, study year, area, principal components and batch. In model 1, PRS and education were mutually adjusted, and model 2 further adjusted for behavioral and metabolic risk factors for CHD. We tested the interactions between PRS and education in model 0 . In these models, PRS was treated as a continuous measure or further divided into six groups from the $12.5^{\text {th }}, 25^{\text {th }}, 50^{\text {th }}, 75^{\text {th }}$ and $87.5^{\text {th }}$ percentiles in order to observe nonlinear associations. Before the interactions between PRS and education were tested, model terms were also introduced for the interactions between education and all the control variables as well as PRS and all the control variables. All analyses were carried out in STATA 14.2 (29). 


\section{RESULTS}

At baseline the participants were aged around 48 years and mostly with basic or vocational education (Table 1). About $31 \%$ of men were current smokers and $35 \%$ drank more than $100 \mathrm{~g}$ ethanol per week; the corresponding figures were $20 \%$ and $10 \%$ among women. The level of metabolic risk factors was relatively high. PRS for CHD was relatively evenly distributed by education, although those with higher education had somewhat lower mean PRS in males (Figure 1; $P$ value for categorical education and PRS men=0.019 and women $P=0.351)$.

Both PRS and education were strongly associated with incident CHD hospitalisation or death among participants free of CHD at baseline (Table 2, model 0). With age as the time-scale and adjusted for sex, study year, region of residence, batch and the ten principal components derived from the genomewide data, those in the highest PRS quartile had a HR of 2.26 (95\% CI: 1.97, 2.59) for incident CHD events compared to those in the lowest PRS quartile. Similarly those with basic education had a HR of $1.58(95 \% \mathrm{CI}: 1.32,1.89)$ as compared to those with tertiary degrees. Both these associations were monotonic over the PRS categories. Incorporating PRS and education in the models simultaneously had relatively little contribution to these excess risks (model 1). Further adjustment for baseline diabetes, smoking, BMI, alcohol use, HDL and total cholesterol and blood pressure attenuated the association of education on CHD events by about $50 \%$ (model 2), but only about $10 \%$ for the association between PRS and CHD events. Finally, we evaluated the interactions between PRS and education; those with basic or vocational education and high PRS had increased risk for CHD events (Figure 2; panel A). Further adjustment for behavioral risk factors attenuated the education main effect but not the interaction effect (Figure 2; panel B). However, overall these interaction effects were rather modest.

\section{DISCUSSION}

Summary of the main results 
Using data on a representative population-based sample of about 26000 Finns recruited from risk factor surveys from 1992 to 2012, we show - consistent with prior studies using this and other samples $(21,30,31)$ - that among participants free of CHD at baseline, PRS for CHD is strongly associated with incident CHD hospitalisation or death. Those in the highest quartile of the risk score compared to those in the lowest quartile were about twice as likely to be hospitalised for or die from CHD. This is comparable to the effect of having basic as opposed to tertiary education. Furthermore, we demonstrated that (i) the PRS for CHD and education are only weakly correlated with each other, (ii) the effects of PRS are thus only weakly mediated by education, and (iii) both associations are to some extent attenuated by adjustment for baseline alcohol, smoking, BMI, cholesterol, blood pressure and diabetes. This attenuation is modest for the PRS but about half for education. Those with basic or vocational training and high PRS had particularly high CHD risk.

Interpretation

PRS and education proved to be two strong and rather distinct determinants of CHD events. The effects of education are well known and expected, and their size is consistent with previous studies (32). More variability in the estimates of PRS on CHD events are observed in the literature. Partly these reflect differences in the PRS measures themselves - with new modifications published at regular intervals - partly that in populations with different ancestry the predictive power of PRS vary (33). The results further demonstrate that the correlation between PRS and education was modest and incorporating both measures in the same regression model led to only modest attenuation of effects. On the one hand, these findings suggest that the effect of PRS on CHD events is not mediated by education, and on the other hand that the effect estimates for education are not explained by underlying genetic risk. Our results are consistent with studies using both school reforms as natural experiments (34) and Mendelian randomization studies (35) that demonstrate robust effects of education on health. Often the effect sizes for education that are obtained from such studies are similar to those obtained in observational research. 
The behavioural and other risk factors were only measured once at baseline. Because of both measurement error and inadequacy of capturing life-time exposure to these risk factors with single measurements, we are underestimating the contribution that these factors have in mediating the effects of both PRS and education on incident CHD events. It is, however, a common finding in the literature that the associations between PRS and CHD are not mediated by these well known behavioural risk factors $(19,29)$. It thus seems likely that genetic risk influences CHD partly through more direct biological pathways that are not associated with behaviours. Overall, the effects of high PRS on CHD represent the combined influences of multiple pathways associated with genetic vulnerability. In thus study approximately $50 \%$ of the association between education and CHD events was attenuated by the inclusion of these risk factors, a finding consistent with earlier studies. Previous evidence also raises the possibility that this is an underestimate. For example, indirect estimates - e.g. based on lung cancer mortality rates - for the contribution of smoking alone indicate that as much as $50 \%$ of socioeconomic differentials in all-cause mortality among men are driven by smoking (36). Also alcohol consumption contributes to levels and trends in social differentials in mortality in many countries (37).

The effects of higher genetic risk on CHD events appear to be slightly exacerbated among those with low education. The motivation to search for gene-enviroment interactions is that environmental social risk factors may activate genes predisposing to CHD. Measures of social position are broad but still objectively measured determinants of CHD, and they are well-known to be associated with CHD risk factors. Previous evidence has shown PRS effect modification with the well-established behavioural CHD risk factors such as smoking, BMI, cholesterol, and blood pressure (19, 22). We adjusted for these factors and observed that while the effects of education on CHD events were attenuated, the tentative interaction could not be attributed to these risk factors alone. Overall, the results indicate that PRS-education interactions are at best modest, but these results need to be validated in future studies. 
Methodological considerations

In Finland - like elsewhere - survey participation rates have declined over time, although our samples have had comparatively high rates also more recently. Additional analyses in supplementary table 2 shows that the results we obtain from the 1992 with the highest response rate (about $75 \%$ ) are very similar to the results from the full sample.

The PRS that we used have been developed in an international multi-center consortium and validated against external CHD events data (21). Supplementary analyses (Supplementary table 3 and supplementary figure 1) show that an alternative PRS for CHD obtained from Tada et al. (27), confirm our main result that PRS for CHD and education predict CHD events independently. Our estimates of the effects of education on CHD events correspond well with similar estimates obtained elsewhere (27). However, as the discovery GWAS studies become larger, new PRSs for CHD will increasingly capture meaningful genetic variation. Our findings on interactiions may thus be provisional in the sense that they may change as more powerful PRSs become available.

Overall, our data represent the Finnish target population well, and the results are likely to have broader generalizability to other high-income countries. However, PRSs have mainly been developed in relatively homogenous populations with European ancestry and generalization to populations with differing ancestries should be carried out with caution (33). Replication of these analyses in other population-based studies are needed to confirm our results, in particular as they relate to the interaction between education and PRS. To further understand these interactions in detail future studies could incorporate information on PRS for education.

\section{Conclusions}

In summary, we show that PRS for CHD and education strongly predict CHD events, and that these effects are to a large extent independent of each other. PRS together with individual social characteristics improve CHD prediction beyond behavioural risks. We further show that the 
association between education and CHD events is strongly attenuated by the well established behavioural and metabolic risk factors. However, it remains partially unclear how the information on improved prediction associated with PRS can help practice as these effects appear to be mostly unrelated to known modifiable behavioral risks. Nevertheless, identification of those with higher genetic risk for CHD in clinical settings may still be useful, because previous evidence indicates, for example, that they may disproportionally benefit from statin treatment to prevent CHD (38). Overall, our results show that when building the next generation of CHD risk estimation tools with genetic data, it is also important to take social factors such as the educational level into account. The results further indicate that observational studies without information on genetic risk for CHD do not provide biased estimates for the association between education and incident CHD events. 
Table 1. Distribution of study population by baseline characteristics, FINRISK Study, Finland, 1992-2015.

\begin{tabular}{|c|c|c|c|c|}
\hline & \multicolumn{2}{|c|}{ Men } & \multicolumn{2}{|c|}{ Women } \\
\hline & $\mathrm{n}$ & $\%$ & $\mathrm{n}$ & $\%$ \\
\hline \multicolumn{5}{|l|}{ PRS quartile } \\
\hline 1 Low & 3138 & 25.6 & 3413 & 24.5 \\
\hline 2 & 3094 & 25.3 & 3460 & 24.8 \\
\hline 3 & 2999 & 24.5 & 3552 & 25.5 \\
\hline 4 High & 3017 & 24.6 & 3530 & 25.3 \\
\hline \multicolumn{5}{|l|}{ Education } \\
\hline Tertiary & 1803 & 14.7 & 2308 & 16.5 \\
\hline Upper secondary & 2597 & 21.2 & 4051 & 29.0 \\
\hline Vocational training & 3661 & 29.9 & 3094 & 22.2 \\
\hline Basic & 4187 & 34.2 & 4502 & 32.3 \\
\hline \multicolumn{5}{|l|}{ Region of residence } \\
\hline North Karelia & 2627 & 21.5 & 2918 & 20.9 \\
\hline North Savonia & 2269 & 18.5 & 2697 & 19.3 \\
\hline Turku/Loimaa & 2389 & 19.5 & 2763 & 19.8 \\
\hline Helsinki/Vantaa & 2747 & 22.4 & 2988 & 21.4 \\
\hline Oulu province & 1739 & 14.2 & 1985 & 14.2 \\
\hline Lapland & 477 & 3.9 & 604 & 4.3 \\
\hline \multicolumn{5}{|l|}{ Smoking } \\
\hline Never smoker & 4984 & 40.7 & 8850 & 63.4 \\
\hline Former smoker & 3447 & 28.1 & 2274 & 16.3 \\
\hline Current smoker & 3817 & 31.2 & 2831 & 20.3 \\
\hline \multicolumn{5}{|l|}{ Alcohol use } \\
\hline Non-drinker & 1611 & 13.2 & 2562 & 18.4 \\
\hline Low moderate drinker ( $<35 \mathrm{~g}$ ethanol/week) & 3317 & 27.1 & 7184 & 51.5 \\
\hline $\begin{array}{l}\text { High moderate drinker (35-100 g } \\
\text { ethanol/week) }\end{array}$ & 3094 & 25.3 & 2809 & 20.1 \\
\hline Heavy drinker (>100 g ethanol per week) & 4226 & 34.5 & 1400 & 10.0 \\
\hline \multirow[t]{2}{*}{ Diabetes } & 885 & 7.2 & 866 & 6.2 \\
\hline & Mean & SD & Mean & SD \\
\hline Age & 48.9 & 13.4 & 48.0 & 13.3 \\
\hline Body mass index $\left(\mathrm{kg} / \mathrm{m}^{2}\right)$ & 27.1 & 4.1 & 26.4 & 5.1 \\
\hline HDL cholesterol (mmol/l) & 1.3 & 0.3 & 1.6 & 0.4 \\
\hline Total cholesterol (mmol/l) & 5.5 & 1.1 & 5.5 & 1.1 \\
\hline Systolic blood pressure (mmHg) & 138.2 & 18.4 & 132.4 & 20.2 \\
\hline Diastolic blood pressure $(\mathrm{mmHg})$ & 83.1 & 11.5 & 78.5 & 10.8 \\
\hline Total & 12248 & & 13955 & \\
\hline
\end{tabular}

PRS, polygenic risk score for coronary heart disease; SD, strandard deviation 
Table 2. Distribution (\%) of the study population and hazard ratios ${ }^{\text {a }}$ for coronary heart disease hospitalisation or death by polygenetic risk score for coronary heart disease and education group (n=26 203), FINRISK study, Finland, 1992-2015.

\begin{tabular}{|c|c|c|c|c|c|c|c|c|c|}
\hline \multirow{2}{*}{$\begin{array}{l}\text { PRS and education } \\
\text { group }\end{array}$} & \multirow[b]{2}{*}{$\%$} & \multicolumn{2}{|c|}{ Model $0^{\mathrm{b}}$} & \multicolumn{2}{|c|}{ Model $0^{b}$} & \multicolumn{2}{|c|}{ Model $1^{c}$} & \multicolumn{2}{|c|}{ Model $2^{d}$} \\
\hline & & $\mathrm{HR}$ & $95 \% \mathrm{Cl}$ & $H R$ & $95 \% \mathrm{Cl}$ & HR & $95 \% \mathrm{Cl}$ & $\mathrm{HR}$ & $95 \% \mathrm{Cl}$ \\
\hline \multicolumn{10}{|l|}{ PRS quartile } \\
\hline 1 Low & 25.0 & 1.00 & Referent & & & 1.00 & Referent & 1.00 & Referent \\
\hline 2 & 25.0 & 1.29 & $1.11,1.49$ & & & 1.29 & $1.12,1.49$ & 1.24 & $1.08,1.44$ \\
\hline 3 & 25.0 & 1.57 & $1.36,1.81$ & & & 1.57 & $1.37,1.81$ & 1.50 & $1.30,1.73$ \\
\hline 4 High & 25.0 & 2.26 & $1.97,2.59$ & & & 2.27 & $1.98,2.61$ & 2.12 & $1.84,2.43$ \\
\hline \multicolumn{10}{|l|}{ Education } \\
\hline Tertiary & 15.7 & & & 1.00 & Referent & 1.00 & Referent & 1.00 & Referent \\
\hline High school & 25.4 & & & 1.11 & $0.91,1.37$ & 1.12 & $0.91,1.38$ & 1.04 & $0.85,1.28$ \\
\hline Vocational training & 25.8 & & & 1.47 & $1.21,1.78$ & 1.49 & $1.23,1.80$ & 1.30 & $1.07,1.57$ \\
\hline Basic & 33.2 & & & 1.58 & $1.32,1.89$ & 1.60 & $1.34,1.91$ & 1.29 & $1.08,1.55$ \\
\hline
\end{tabular}

$\mathrm{Cl}$, confidence interval; $\mathrm{HR}$, hazard ratio; PRS, polygenetic risk score for coronary heart disease

${ }^{\text {a }}$ All models adjusted for sex, region of residence, calendar year, study batch and principal components

${ }^{\mathrm{b}}$ Hazard ratios for PRS and education from separate models

${ }^{\mathrm{c}}$ PRS and education mutually adjusted

${ }^{d}$ Adjusted for smoking, alcohol use, body mass index, HDL and total cholesterol, blood pressure and diabetes 


\section{References}

1. Lozano R, Naghavi M, Foreman K, et al. Global and regional mortality from 235 causes of death for 20 age groups in 1990 and 2010: a systematic analysis for the Global Burden of Disease Study 2010. The lancet. 2012;380(9859):2095-2128.

2. Mackenbach JP, Stirbu I, Roskam A-JR, et al. Socioeconomic inequalities in health in 22 European countries. New England Journal of Medicine. 2008;358(23):2468-2481.

3. Huisman M, Kunst AE, Bopp M, et al. Educational inequalities in cause-specific mortality in middleaged and older men and women in eight western European populations. The Lancet. 2005;365(9458):493-500.

4. Kunst AE, Leon DA, Groenhof F, et al. Occupational class and cause specific mortality in middle aged men in 11 European countries: comparison of population based studiesCommentary: Unequal inequalities across Europe. Bmj. 1998;316(7145):1636-1642.

5. Kilpi F, Silventoinen K, Konttinen H, et al. Early-life and adult socioeconomic determinants of myocardial infarction incidence and fatality. Social Science \& Medicine. 2017;177:100-109.

6. McLaren L. Socioeconomic status and obesity. Epidemiologic reviews. 2007;29(1):29-48.

7. Silventoinen K, Pankow J, Jousilahti P, et al. Educational inequalities in the metabolic syndrome and coronary heart disease among middle-aged men and women. International Journal of Epidemiology. 2005;34(2):327-334.

8. Elks CE, Den Hoed M, Zhao JH, et al. Variability in the heritability of body mass index: a systematic review and meta-regression. Frontiers in endocrinology. 2012;3:29.

9. Silventoinen K, Jelenkovic A, Sund R, et al. Differences in genetic and environmental variation in adult BMI by sex, age, time period, and region: an individual-based pooled analysis of 40 twin cohorts. The American journal of clinical nutrition. 2017;106(2):457-466.

10. Evans A, van Baal GCM, McCarron P, et al. The genetics of coronary heart disease: the contribution of twin studies. Twin Research and Human Genetics. 2003;6(5):432-441.

11. Benyamin B, Sørensen TIA, Schousboe K, et al. Are there common genetic and environmental factors behind the endophenotypes associated with the metabolic syndrome? Diabetologia. 2007;50(9):18801888.

12. Marenberg ME, Risch N, Berkman LF, et al. Genetic susceptibility to death from coronary heart disease in a study of twins. New England Journal of Medicine. 1994;330(15):1041-1046.

13. Zdravkovic S, Wienke A, Pedersen NL, et al. Heritability of death from coronary heart disease: a 36year follow-up of 20966 Swedish twins. Journal of internal medicine. 2002;252(3):247-254.

14. Silventoinen K, Hjelmborg J, Möller S, et al. Family aggregation of cardiovascular disease mortality: a register-based prospective study of pooled Nordic twin cohorts. International journal of epidemiology. 2017;46(4):1223-1229.

15. Nikpay M, Stewart AF, McPherson R. Partitioning the heritability of coronary artery disease highlights the importance of immune-mediated processes and epigenetic sites associated with transcriptional activity. Cardiovascular research. 2017;113(8):973-983. 
16. Hou K, Burch KS, Majumdar A, et al. Accurate estimation of SNP-heritability from biobank-scale data irrespective of genetic architecture. Nature genetics. 2019;1.

17. Nikpay M, Goel A, Won H-H, et al. A comprehensive 1000 Genomes-based genome-wide association meta-analysis of coronary artery disease. Nature genetics. 2015;47(10):1121-1130.

18. Global Lipids Genetics Consortium, Willer CJ, Schmidt EM, et al. Discovery and refinement of loci associated with lipid levels. Nature Genetics. 2013;45:1274-1283.

19. Khera AV, Chaffin M, Wade KH, et al. Polygenic prediction of weight and obesity trajectories from birth to adulthood. Cell. 2019;177(3):587-596.

20. Thanassoulis G, Peloso GM, Pencina MJ, et al. A genetic risk score is associated with incident cardiovascular disease and coronary artery calcium: the Framingham Heart Study. Circulation: Genomic and Precision Medicine. 2012;5(1):113-121.

21. Abraham G, Havulinna AS, Bhalala OG, et al. Genomic prediction of coronary heart disease. European heart journal. 2016;37(43):3267-3278.

22. Ahmad S, Rukh G, Varga TV, et al. Gene $\times$ Physical Activity Interactions in Obesity: Combined Analysis of 111,421 Individuals of European Ancestry. PLOS Genetics. 2013;9(7):e1003607.

23. Silventoinen K, Hasselbalch AL, Lallukka T, et al. Modification effects of physical activity and protein intake on heritability of body size and composition-. The American journal of clinical nutrition. 2009;90(4):1096-1103.

24. Tyrrell J, Wood AR, Ames RM, et al. Gene-obesogenic environment interactions in the UK Biobank study. Int J Epidemiol. 2017;46(2):559-575.

25. Young AI, Wauthier F, Donnelly P. Multiple novel gene-by-environment interactions modify the effect of FTO variants on body mass index. Nat Commun. 2016;7.

26. Borodulin K, Tolonen H, Jousilahti P, et al. Cohort Profile: The National FINRISK Study. International journal of epidemiology. 2017;47(3):696-696i.

27. Tada H, Melander O, Louie JZ, et al. Risk prediction by genetic risk scores for coronary heart disease is independent of self-reported family history. European heart journal. 2016;37(6):561-567.

28. Pajunen P, Koukkunen H, Ketonen M, et al. The validity of the Finnish Hospital Discharge Register and Causes of Death Register data on coronary heart disease. European Journal of Cardiovascular Prevention \& Rehabilitation. 2005;12(2):132-137.

29. StataCorp. Stata Statistical Software: Release 14. College Station, TX: StataCorp LP. 2015;

30. Mars N, Koskela JT, Ripatti P, et al. Polygenic and clinical risk scores and their impact on age at onset and prediction of cardiometabolic diseases and common cancers. Nature Medicine. 2020;26(4):549_ 557.

31. Ripatti S, Tikkanen E, Orho-Melander M, et al. A multilocus genetic risk score for coronary heart disease: case-control and prospective cohort analyses. The Lancet. 2010;376(9750):1393-1400.

32. de Gelder R, Menvielle G, Costa G, et al. Long-term trends of inequalities in mortality in 6 European countries. International journal of public health. 2017;62(1):127-141.

33. Martin AR, Kanai M, Kamatani Y, et al. Clinical use of current polygenic risk scores may exacerbate health disparities. Nature genetics. 2019;51(4):584-591. 
34. Lager ACJ, Torssander J. Causal effect of education on mortality in a quasi-experiment on 1.2 million Swedes. Proc Natl Acad Sci U S A. 2012;109(22):8461-8466.

35. Tillmann T, Vaucher J, Okbay A, et al. Education and coronary heart disease: mendelian randomisation study. BMJ. 2017;358.

36. Martikainen P, Ho J, Preston S, et al. The changing contribution of smoking to educational differences in life expectancy: indirect estimates for Finnish men and women from 1971 to 2010. J Epidemiol Community Health. 2013;67(3):219-224.

37. Mackenbach JP, Bopp M, Deboosere P, et al. Determinants of the magnitude of socioeconomic inequalities in mortality: A study of 17 European countries. Health \& place. 2017;47:44-53.

38. Natarajan P, Young R, Stitziel NO, et al. Polygenic risk score identifies subgroup with higher burden of atherosclerosis and greater relative benefit from statin therapy in the primary prevention setting. Circulation. 2017;135(22):2091-2101. 

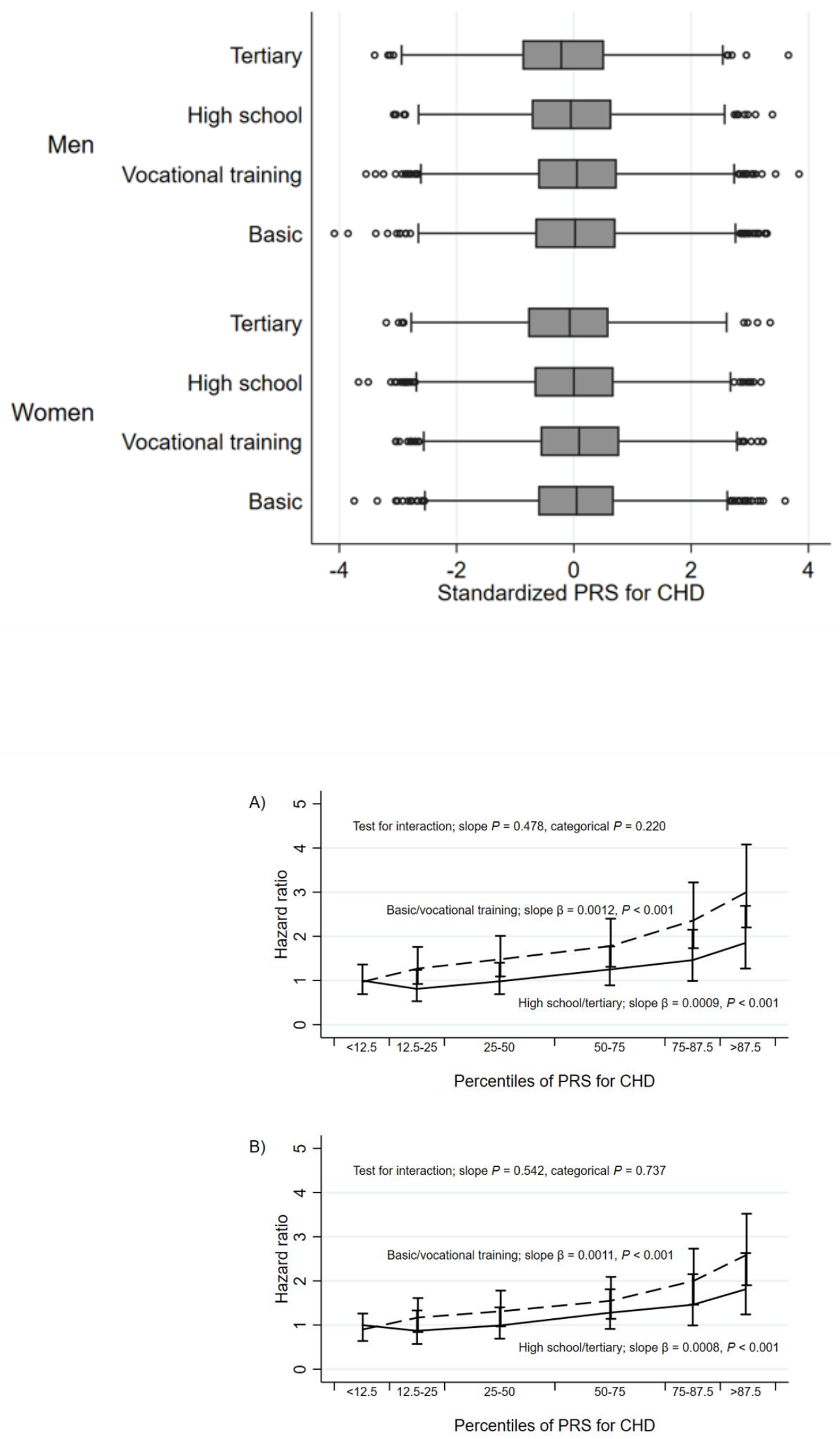

High school/tertiary $\_$Basic/vocational training - - 